PROCEEDINGS OF THE

AMERICAN MATHEMATICAL SOCIETY

Volume 124, Number 3, March 1996

\title{
BANACH SPACES THAT ADMIT SUPPORT SETS
}

\author{
J. M. BORWEIN AND J. D. VANDERWERFF
}

(Communicated by Dale Alspach)

\begin{abstract}
It is shown that the existence of a closed convex set all of whose points are properly supported in a Banach space is equivalent to the existence of a certain type of uncountable ordered one-sided biorthogonal system. Under the continuum hypothesis, we deduce that this notion is weaker than the existence of an uncountable biorthogonal system.
\end{abstract}

\section{INTRODUCTION}

We will say a closed convex set $C$ in a Banach space $X$ is a support set if for every $z \in C$ there is a $\phi \in X^{*}$ such that $\phi(z)=\inf _{C} \phi<\sup _{C} \phi$. It is well known that no such set can exist in a separable Banach space, and Rolewicz asked whether such a set must exist in a nonseparable space. The work of Kutzarova $([\mathrm{K}])$, Lazar $([\mathrm{L}])$ and Montesinos $([\mathrm{M}])$ demonstrates that certain nonseparable spaces have such sets. A common thread in these results is that the authors observe Banach spaces with uncountable biorthogonal systems have support sets, and then they demonstrate various classes of Banach spaces have such systems. Lazar additionally considered $C(\Omega)$ spaces where $\Omega$ is a compact Hausdorff space. In particular, [L, Theorem 2] shows $C(\Omega)$ admits a support set provided $\Omega$ is nonseparable or $\Omega$ admits a closed non- $G_{\delta}$ set.

It is a straightforward topological exercise to show that a compact Hausdorff space $\Omega$ has no closed non- $G_{\delta}$ set if and only if it is hereditarily Lindelöf. One can thus rephrase Lazar's result as: $C(\Omega)$ has a support set if $\Omega$ has a closed nonseparable subspace or $\Omega$ has a non-Lindelöf subspace. Using some standard results for compact Hausdorff spaces, this is equivalent to saying that support sets exist in $C(\Omega)$ unless $\Omega$ is simultaneously hereditarily separable and hereditarily normal (see, [E], p. 228, 3.12.9(e)). Observe that this does not cover all nonseparable $C(\Omega)$ spaces. For example one can check that the double arrow space $\Omega$ is hereditarily Lindelöf and hereditarily separable, while $C(\Omega)$ has an uncountable biorthogonal system; see e.g. [FiG, Example II.5(1)].

However, it was unknown if Lazar's ([L, Theorem 2]) conditions on $\Omega$ actually imply the existence of an uncountable biorthogonal system in $C(\Omega)$ (the proof of

Received by the editors May 24, 1994

1991 Mathematics Subject Classification. Primary 46B03, 46B20.

Key words and phrases. Proper support point, support set, uncountable biorthogonal system, convex set.

The first author's research was supported in part by an NSERC research grant and by the Shrum endowment.

The second author is a NSERC postdoctoral fellow. 
[L, Theorem 2(b)] shows $C(\Omega)$ has such a system if $\Omega$ is nonseparable and hereditarily Lindelöf). More generally, there was no known example of a Banach space with a support set but no uncountable biorthogonal system. Under the continuum hypothesis, Proposition 1 provides such an example. This also shows the necessity for weakening the notion of a biorthogonal system in order to describe spaces with support sets as is done in Theorem 4.

\section{A CHARACTERIZATION OF SPACES ADMITTING SUPPORT SETS}

It is asked in [FiG, Question IV.2] whether Kunen's $C(\Omega)$ space (see [N, pp. 1123-1129]) has a support set even though it has no uncountable biorthogonal system; note that Kunen's construction uses the continuum hypothesis. We begin by showing Kunen's space has a rather nice support set by strengthening and simplifying Lazar's result on non- $G_{\delta}$ closed sets ([L, Theorem 2(a)]).

Proposition 1. (a) If $\Omega$ is a compact Hausdorff space and $F$ is a closed non- $G_{\delta}$ set, then $K=\{f \in C(\Omega): f(F)=\{0\}, f \geq 0\}$ is a support cone (i.e., $t K \subset K$ for all $t \geq 0)$ and $K \cap\left\{f:\|f\|_{\infty} \leq r\right\}$ is a support set for any $r>0$.

(b) Kunen's $C(\Omega)$ space has a support cone $K$ such that $K-K=C(\Omega)$.

Proof. (a) Let $f \in K$. Because $F$ is not a $G_{\delta}$ set, $F$ is a proper subset of $f^{-1}(0)$. Thus we choose $p \notin F$ with $f(p)=0$. Now consider $\delta_{p}$ (the point mass measure at $p)$, then $\delta_{p}(f)=f(p)=0=\inf _{K} \delta_{p}$, while $\sup _{K} \delta_{p}=\infty$ (by Tietze); in the second case nothing changes except the sup of $\delta_{p}$, which is now $r>0$.

(b) $\Omega$ is the one-point compactification $S \cup\{\infty\}$ where $S$ is a non-Lindelöf locally compact Hausdorff space (see [N, Theorem 7.1]). Hence $\{\infty\}$ is not a $G_{\delta}$ set or else $S$ would be $\sigma$-compact and Lindelöf. We now let $K=\{t(f+1): f(\infty)=$ $0, f \geq 0, t \geq 0\}$. It is easy to check that $K-K=C(\Omega)$ and that $K$ is a support cone (consider $\delta_{p}-\delta_{\infty}$ as in the argument of (a), or see Fact 2 for a more general approach).

Fact 2. If $X$ has a support set, then $X \oplus \mathbb{R}$ has a support cone.

Proof. Let $C$ be a support set in $X$. Define $K \subset X \oplus \mathbb{R}$, by $K=\{t(x, 1): x \in C, t \geq$ $0\}$. Consider $t_{0}\left(x_{0}, 1\right) \in K$ and choose $\phi_{0} \in X^{*}$ such that $\phi_{0}$ properly supports $C$ at $x_{0}$. It is easy to check that $\left(\phi_{0},-\phi_{0}\left(x_{0}\right)\right)$ supports $K$ at $t_{0}\left(x_{0}, 1\right)$.

We turn to the task of finding a notion that characterizes spaces with support sets. For the remainder of this note, we will let $X$ be a Banach space and $Y \subset X^{*}$ be a total norm closed subspace. We let $\tau$ denote any topology on $X$ such that $\tau$ is either the weak topology induced by $Y$, the norm topology or any topology intermediate to them. A closed convex set $C$ is called a $Y$-support set if for every $z \in C$ there is a $\phi \in Y$ such that $\phi(z)=\inf _{C} \phi<\sup _{C} \phi$. In the case $Y=X^{*}$, we will continue to say $C$ is a support set; if $C$ is in a dual Banach space $X^{*}$ and $Y=X$, then we shall say $C$ is a $w^{*}$-support set.

Lemma 3. (a) If $C$ is a $Y$-support set and $\Lambda \in X^{*}$ is such that $\inf _{C} \Lambda<\alpha<$ $\sup _{C} \Lambda$, then $C_{\alpha}:=\Lambda^{-1}(\alpha) \cap C$ is a $Y$-support set. If moreover, $\Lambda \in Y$, then $\Lambda^{-1}(0)$ admits a $Y_{1}$-support set where $Y=Y_{1} \oplus \operatorname{span}\{\Lambda\}$.

(b) If $X$ has a support set, then any subspace of finite codimension in $X$ has a support set. 
Proof. (a) Let $x_{0} \in C_{\alpha}$ be arbitrary. We wish to show $x_{0}$ is properly supported in $C_{\alpha}$ by a functional in $Y$, so we fix $\phi \in Y$ such that $\phi\left(x_{0}\right)=\inf _{C} \phi<$ $\sup _{C} \phi$. Now choose $\bar{x} \in C$ such that $\phi(\bar{x})>\phi\left(x_{0}\right)$. If $\bar{x} \in C_{\alpha}$ there is nothing further to do. So we suppose $\Lambda(\bar{x}) \neq \alpha$. If $\Lambda(\bar{x})>\alpha$, choose $y \in C$ such that $\Lambda(y)<\alpha$. Now there is a convex combination $\bar{x}_{\alpha}=t \bar{x}+(1-t) y$ with $0<t<1$ and $\bar{x}_{\alpha} \in C_{\alpha}$; clearly $\phi\left(\bar{x}_{\alpha}\right)>\phi\left(x_{0}\right)$. If $\Lambda(\bar{x})<\alpha$, one chooses $y \in C$ with $\Lambda(y)>\alpha$ and proceeds as above to complete the proof of (a). To prove the moreover part, we continue by writing $\phi=\phi_{1}+\beta \Lambda$ where $\phi_{1} \in Y_{1}$. Since $\beta \Lambda$ is constant on $\Lambda^{-1}(0)$, it follows that $\phi_{1}$ properly supports $C_{\alpha}$ at $x_{0}$. Translating $C_{\alpha}$ to $\Lambda^{-1}(0)$ completes the proof.

(b) Since all hyperplanes in $X$ are isomorphic, it follows from (a) that they must all have support sets. The proof is completed by induction.

Let $\omega_{1}$ denote the first uncountable ordinal. We will call a collection

$$
\left\{x_{\alpha}, f_{\alpha}\right\}_{1 \leq \alpha<\omega_{1}} \subset X \times X^{*}
$$

a semibiorthogonal system if $f_{\mu}\left(x_{\alpha}\right)=0$ for all $\alpha<\mu, f_{\alpha}\left(x_{\alpha}\right)=1$ and $f_{\mu}\left(x_{\alpha}\right) \geq 0$ for all $\alpha$; this is a weakening of an uncountable biorthogonal system where one has $f_{\mu}\left(x_{\beta}\right)=0$ for all $\mu \neq \alpha$.

Theorem 4. For a Banach space $X$ and $Y$ a total subspace of $X^{*}$, the following are equivalent.

(a) There is a semibiorthogonal system $\left\{x_{\alpha}, f_{\alpha}\right\}_{1 \leq \alpha<\omega_{1}} \subset X \times Y$.

(b) $X$ has a $\tau$-sequentially closed $Y$-support cone for all topologies $\tau$ as described above.

(c) $X$ has a norm closed $Y$-support cone.

(d) $X$ has a norm closed (bounded) $Y$-support set.

Proof. (a) $\Rightarrow(\mathrm{b})$ and (d): This is essentially as in [L, Theorem 1]. Let $K$ be the $\tau$-sequential closure of the convex cone $K^{\circ}$ generated by $\left\{x_{\alpha}\right\}_{1 \leq \alpha<\omega_{1}}$. If $k \in K$, then we choose $k_{n} \in K^{\circ}$ converging $\tau$ to $k$. Now $k_{n} \in \operatorname{span}\left(\left\{x_{\alpha}\right\}_{1 \leq \alpha \leq \mu}\right)$ for some countable ordinal $\mu$ and for all $n$. Thus $f_{\mu+1}\left(k_{n}\right)=0$ for all $n$ and so $f_{\mu+1}(k)=0$ by the properties of $\tau$ which also ensure $\inf _{K} f_{\mu+1} \geq 0$. Now $f_{\mu+1}\left(x_{\mu+1}\right)=1$, and so $K$ is properly supported by $f_{\mu+1}$ at $k$. Also, after normalizing the system so $\left\|x_{\alpha}\right\|=1$ for all $\alpha$, the same proof shows the $\tau$-sequentially closed convex hull of $\left\{x_{\alpha}\right\}_{1 \leq \alpha<\omega}$ is a bounded $Y$-support set.

(b) $\Rightarrow$ (c): This follows by letting $\tau$ be the norm topology.

(c) $\Rightarrow$ (a): Let $K$ be a norm closed $Y$-support cone. Fix $x_{0} \in K$ and choose $f_{1} \in Y$ such that $f_{1}\left(x_{0}\right)=\inf _{K} f_{1}<\sup _{K} f_{1}$. Note $f_{1}\left(x_{0}\right)$ must be 0 , since $K$ is a cone. By scaling $f_{1}$ if necessary, we choose $x_{1} \in S_{X} \cap K$ such that $f_{1}\left(x_{1}\right)=1$. Suppose $\mu$ is a countable ordinal and $\left\{x_{\alpha}, f_{\alpha}\right\} \subset S_{X} \times Y$ have been chosen for $\alpha<\mu$ so that $f_{\alpha}\left(x_{\alpha}\right)=1, f_{\beta}\left(x_{\alpha}\right)=0$ for $\alpha<\beta<\mu$ and $f_{\beta}\left(x_{\alpha}\right) \geq 0$ for all $\alpha, \beta<\mu$. Let $\bar{x}=\sum_{\alpha<\mu} c_{\alpha} x_{\alpha}$ where $\sum_{\alpha<\mu} c_{\alpha}=1$ and $c_{\alpha}>0$ for each $\alpha$. Choose $f_{\mu} \in Y$ such that $f_{\mu}(\bar{x})=\inf _{K} f_{\mu}<\sup _{K} f_{\mu}$. Now $f_{\mu}(k) \geq 0$ for all $k \in K$, and since each $c_{\alpha}>0$, we have $f_{\mu}\left(x_{\alpha}\right)=0$ for each $\alpha<\mu$. To complete the inductive step, scaling $f_{\mu}$ if necessary we choose $x_{\mu} \in K \cap S_{X}$ such that $f_{\mu}\left(x_{\mu}\right)=1$.

$(\mathrm{d}) \Rightarrow(\mathrm{c})$ : Suppose $C$ is a $Y$-support set in $X$, there is no need to assume boundedness. Now take $\Lambda \in Y$ such that $\inf _{C} \Lambda<\sup _{C} \Lambda$ and write $Y=Y_{1} \oplus$ 
$\operatorname{span}\{\Lambda\}$. According to Lemma $3(\mathrm{a}), \Lambda^{-1}(0)$ admits a $Y_{1}$-support set $C$. Fix $\bar{x} \in X$ such that $\Lambda(\bar{x})=1$, and let

$$
K=\{t(z+\bar{x}): z \in C, t \geq 0\} .
$$

Fix $t_{0}\left(z_{0}+\bar{x}\right) \in K$, choose $\phi_{0} \in Y_{1}$ such that $\phi_{0}$ properly supports $C$ at $z_{0}$ and then choose $\alpha_{0}$ such that $\left(\phi_{0}+\alpha_{0} \Lambda\right)(\bar{x})=-\phi\left(z_{0}\right)$. Now $\left(\phi_{0}+\alpha_{0} \Lambda\right)(t(z+\bar{x}))=$ $t\left[\phi_{0}(z)-\phi_{0}\left(z_{0}\right)\right]$ and so one can easily check that $\phi+\alpha_{0} \Lambda$ properly supports $K$ at $t_{0}\left(z_{0}+\bar{x}\right)$.

As a consequence of this characterization, we now show that Kunen's space is distinguished from spaces admitting uncountable biorthogonal systems by $w^{*}$ support sets.

Corollary 5. (a) If $X$ has an uncountable biorthogonal system, then $X^{*}$ has a $w^{*}$-sequentially closed $w^{*}$-support set.

(b) If $X$ is Kunen's $C(\Omega)$ space, then $X^{*}$ has no norm closed $w^{*}$-support set.

Proof. Theorem 4 shows that (a) is true. To prove (b), suppose $C$ is a $w^{*}$-support set. Then by Theorem 4 , we have a semibiorthogonal system $\left\{\phi_{\alpha}, x_{\alpha}\right\}_{1 \leq \alpha<\omega_{1}} \subset$ $X^{*} \times X$. Hence $\phi_{\alpha}\left(x_{\beta}\right)=0$ for all $\beta>\alpha$ and so $x_{\alpha} \notin \overline{\operatorname{conv}}\left(\left\{x_{\beta}: \beta>\alpha\right\}\right)$. However Kunen's space cannot have such a right-separated family; see [N, p. 1128].

Remark 6. (a) The $w^{*}$-closure of a $w^{*}$-support cone in $X^{*}$ need not be a support cone. Indeed, one can check that the $w^{*}$-closure of the cone of nonnegative countably supported elements in $\ell_{\infty}(\Gamma)$ for $\Gamma$ uncountable is not a support set. However, such $\ell_{\infty}(\Gamma)$ spaces have $w^{*}$-closed $w^{*}$-support sets as does any space which is the second dual of a space containing an uncountable biorthogonal system $\left\{x_{\alpha}, f_{\alpha}\right\}_{\alpha \in A}$ such that $\overline{\operatorname{conv}}\left(\left\{x_{\alpha}\right\}_{\alpha \in A}\right)$ is weakly compact.

(b) Since $\Omega$ is scattered in Kunen's $C(\Omega)$ (see [N, Theorem 7.7]), its dual is $\ell_{1}(\Omega)$. One can check that the positive cone in $\ell_{1}(\Omega)$ is a support set closed in the weak star topology induced by $C(\Omega)$. However, by Corollary 5 , it cannot be a $w^{*}$-support set.

(c) The deep structural results of [FG] and [S] show that every nonseparable dual space has an uncountable biorthogonal system. Using the fact that such systems are lifted by quotients ([FiG, p. 90], [M, p. 67]) one has that every Banach space with a nonseparable dual space as a quotient has such a system. However, it is still unknown whether every nonseparable Banach space has a support set. The specific case of nonseparable $C(\Omega)$ spaces is also open.

(d) To construct a $Y$-support set, the following system that appears slightly weaker than a semibiorthogonal system suffices: $\left\{x_{\alpha}, f_{\alpha}, a_{\alpha}\right\}_{1 \leq \alpha<\omega_{1}} \subset X \times Y \times \mathbb{R}$ satisfying $f_{\alpha}\left(x_{\beta}\right)=a_{\alpha}$ for all $\beta<\alpha, f_{\alpha}\left(x_{\alpha}\right)>a_{\alpha}$ and $f_{\alpha}\left(x_{\beta}\right) \geq a_{\alpha}$ for $\beta \geq \alpha$ (cf. Theorem 4).

\section{REFERENCES}

[E] R. Engleking, Outline of General Topology, North-Holland, Amsterdam, 1989.

[FG] M. Fabian and G. Godefroy, The dual of every Asplund space admits a projectional resolution of identity, Studia Math. 91 (1988), 141-151. MR 90b:46032

[FiG] C. Finet and G. Godefroy,, Biorthogonal systems and big quotient spaces, Contemp. Math., vol. 85, Amer. Math. Soc., Providence, RI, 1989, pp. 87-110. MR 90f: 46022

[K] D. Kutzarova, Convex sets containing only support points in Banach spaces with an un countable minimal system, C. R. l'Acad. Bulg. Science 39 (1986), 13-14. MR 88f:46041 
[L] A. J. Lazar, Points of support for closed convex sets, Illinois J. Math. 25 (1981), 302-305. MR 82h: 46029

[M] V. Montesinos, Solution to a problem of S. Rolewicz, Studia Math. 81 (1985), 65-69. MR 87c: 46024

[N] S. Negrepontis, Banach spaces and topology, Handbook of Set-Theoretic Topology (1984), 1045-1142. MR 86i:46018

[S] C. Stegall, The Radon-Nikodým property in conjugate Banach spaces, Trans. Amer. Math. Soc. 206 (1975), 213-223. MR 51:10581

Department of Mathematics and Statistics, Simon Fraser University, Burnaby, British Columbia, Canada V5A 1S6

Current address, J. D. Vanderwerff: Department of Mathematics, Walla Walla College, College Place, Washington 99324 